\title{
Determinação dos campos de velocidades de escoamentos bifásicos usando PIV-PTV
}

\section{Determination of velocity fields of two-phase flows using PIV-PTV}

\author{
Rui Aleixo', Elsa Carvalho', Maria Manuela C.L. Lima ${ }^{3}$ e Rui M. L. Ferreira ${ }^{4}$ \\ ${ }^{1}$ Faculdade de Engenharia da Universidade do Porto, Porto, Portugal, raleixo@fe.up.pt \\ ${ }^{2}$ Faculdade de Engenharia da Universidade do Porto, Porto, Portugal, elsac@fe.up.pt \\ ${ }^{3}$ Escola de Engenharia da Universidade do Minho, Guimarães, Portugal, mmlima@civiluminho.pt \\ ${ }^{4}$ CERIS Instituto Superior Técnico, Lisboa, Portugal, ruimferreira@tecnico.ulisboa.pt
}

RESUMO: No domínio da hidráulica fluvial, é fundamental conhecer o campo de velocidades das fases sólida (sedimentos) e líquida (água). A partir do campo de velocidades instantâneas é possível quantificar diversas variáveis incluindo intensidades da turbulência e tensões de corte e identificar zonas de separação, recirculação, etc.

O desenvolvimento de técnicas óticas permitiu o desenvolvimento de instrumentos de medição baseados na aquisição e no processamento de imagens, nomeadamente o PIV (particle image velocimetry) e o PTV (particle tracking velocimetry).

Neste artigo, apresenta-se um algoritmo que combina ambas as técnicas, PIV e PTV, permitindo assim, juntar os pontos fortes de cada uma das técnicas e possibilitando a medição simultânea do campo de velocidades da água e dos sedimentos e estudar mais detalhadamente a interação fluido-sedimentos.

As vantagens e limitações do algoritmo proposto são exploradas e discutidas com recurso a um conjunto de experiências laboratoriais.

Palavras-chave: Velocimetria por imagem de partículas, velocimetria por seguimento de partículas, escoamento bifásico, rutura de barragem, escoamento transiente.

ABSTRACT: In the domain of fluvial hydraulics, it is critical to know the velocity field of both solid (sediments) and liquid phases (water). From the velocity field it is possible to determine different variables such as shear stresses, identify recirculation regions, etc.

The development of optical techniques allowed for measurement techniques based on image acquisition and processing, namely PIV (particle image velocimetry) and PTV (particle tracking velocimetry).

In this paper an algorithm combining both PIV and PTV techniques is presented, allowing in this way to profit from each technique strong points and allowing the simultaneous measurement of the water and sediment layer velocity fields and to obtain a better know how of the fluid-sediment interaction.

The advantages and limitation of the proposed techniques are explored and discussed by means of a laboratory experiment. Keywords: Particle image Velocimetry, particle tracking velocimetry, two-phase flow, dam-break, transient flow. 


\section{VELOCIMETRIA POR IMAGEM DE PARTÍCULAS (PIV)}

A velocimetria por imagem de partículas (Particle Image Velocimetry, PIV na sigla inglesa) é uma técnica de medição baseada na aquisição e processamento de imagens de um fluido em movimento ao qual foramadicionadas previamente partículas traçadoras. Imagens consecutivas, separadas por um breve intervalo de tempo, $\mathrm{d} t$, são divididas em pequenas áreas designadas por áreas de interrogação. Através da correlação de tons de cinzento de áreas de interrogação, é possível determinar o deslocamento representativo associado ao conjunto de partículas de cada área. É necessário pelo menos um par de imagens para realizar a técnica PIV.

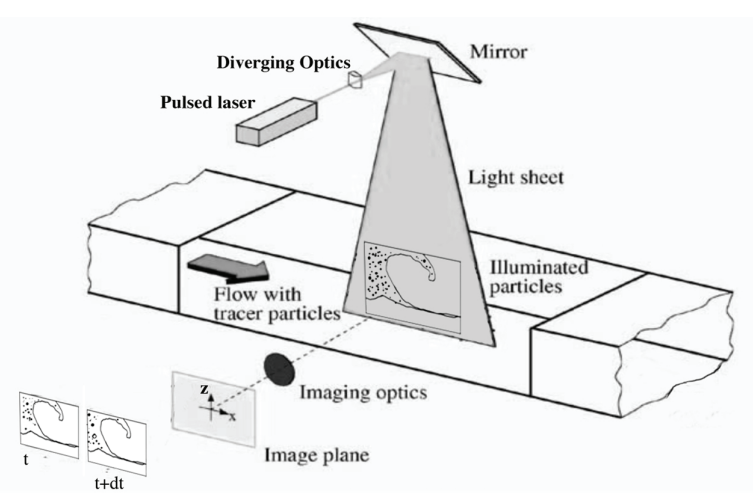

Figura 1. Esquema da técnica PIV. Uma folha laser é gerada a partir de um feixe laser por meio de uma lente divergente (cilíndrica). Esta folha laser é depois projetada no escoamento iluminando uma secção bem definida.

A técnica PIV teve o seu início nos anos 80 do século passado (Meynart, 1982) e é atualmente uma técnica estabelecida e comprovada (Adrian, 2005; Raffel et al., 2007; Adrian e Westerweel, 2011).

Desenvolvimentos notáveis desta técnica foram os de Keane e Adrian (1992) que primeiro consideraram o uso da função de correlação aplicada a imagens sucessivas e a deformação iterativa das áreas de interrogação proposta por Scarano e Riethmuller (2000). O facto de ser baseada na operação de correlação e esta última ser implementada através do algoritmo FFT, faz da técnica PIV uma técnica bastante eficiente do ponto de vista computacional. O esquema da Figura 1 ilustra a técnica de PIV.

A técnica PIV requer uma concentração elevada de partículas traçadoras, existindo um limite empírico de pelo menos 4 partículas na mais pequena janela de interrogação considerada (Raffel et al.,2007; Adrian e Westerweel, 2011).

$\mathrm{O}$ algoritmo de PIV usado neste trabalho foi o MatPIV 1.7, implementado em Matlab e disponível no seguinte endereço: www.mn.uio.no/math/ english/people/aca/jks/matpiv/.

\section{VELOCIMETRIA POR SEGUIMENTO DE PARTÍCULAS (PTV)}

A velocimetria por seguimento de Partículas (Particle Tracking Velocimetry, PTV na sigla inglesa) é uma técnica que, como o nome indica, se baseia no seguimento de partículas traçadoras individuais previamente dispersas no escoamento como indicado na Figura 2. É, portanto, uma técnica lagrangeana. Esta técnica consiste em identificar partículas traçadoras e segui-las no tempo através de um algoritmo de correspondência. A posição inicial e final das partículas identificadas e bem correspondidas é memorizada e utilizada para a determinação da velocidade de cada partícula traçadora a partir da expressão:

$$
\nu=\frac{d x}{d t}
$$

Na equação anterior $v$ representa ovector velocidade velocidade, $d x$ é o vetor deslocamento e $d t$ é o intervalo de tempo entre imagens consecutivas.
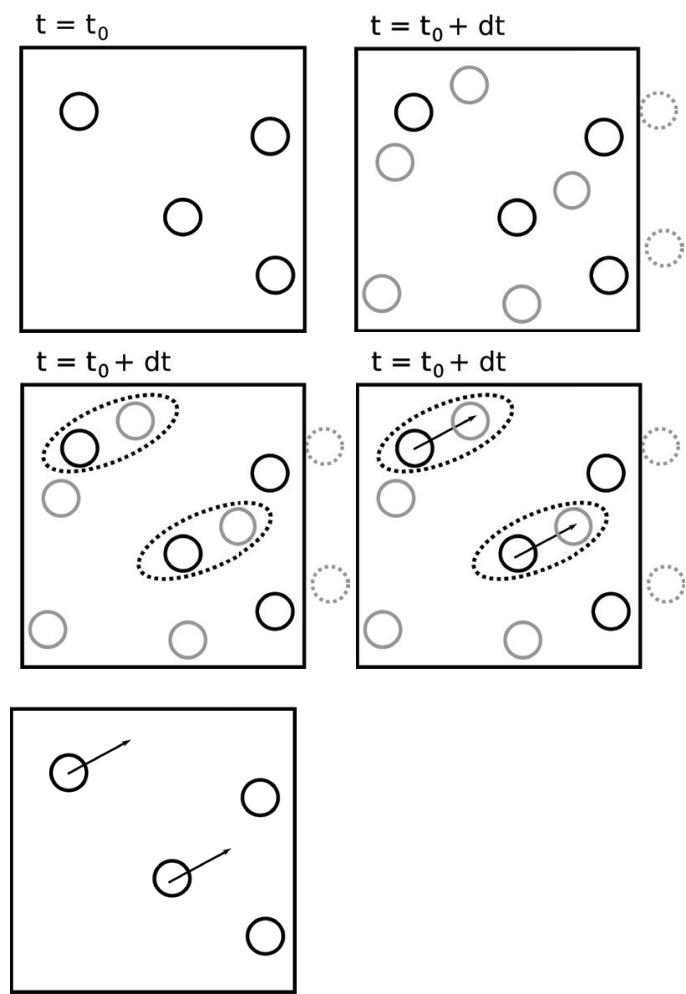

Figura 2. Esquema da técnica PTV. Duas imagens consecutivas obtidas em t0 e t0+dte a determinação da correspondência entre partículas. $O$ vetor deslocamento é determinado pela diferença entre as posições iniciais e finais das partículas. Finalmente o vetor velocidade de cada partícula obtém-se dividindo o vetor deslocamento pelo intervalo de tempo $d t$.

Contrariamente ao PIV, a técnica PTV não é baseada numa operação fundamental. Isto faz com que diversos métodos de seguimento de partículas 
tenham sido propostos ao longo dos anos por diferentes autores. Por outro lado, a operação partícula-a-partícula faz com que os algoritmos de PTV sejam, regra geral, computacionalmente muito pesados, contudo, realizáveis para concentrações pequenas de partículas.

Neste artigo foi utilizado o algoritmo de PTV proposto por Capart et al. (2002), baseado na tesselação Voronoï do espaço. Este algoritmo considera que cada partícula detetada é o centro de um polígono de Voronoï. Dado um polígono de Voronoï, é possível definir a chamada estrela de Voronoï, através da união dos centros dos polígonos vizinhos, conforme ilustrado na Figura 3. O critério para correspondência entre polígonos de Voronoï, $P_{n}, P_{m}$, é obtida a partir da distância entre as extremidades das respetivas estrelas de Voronoï:

$$
\operatorname{match}\left(P_{n}, P_{m}\right)=\min \left(\operatorname{dist}\left(S_{n}, S_{m}\right)\right)
$$
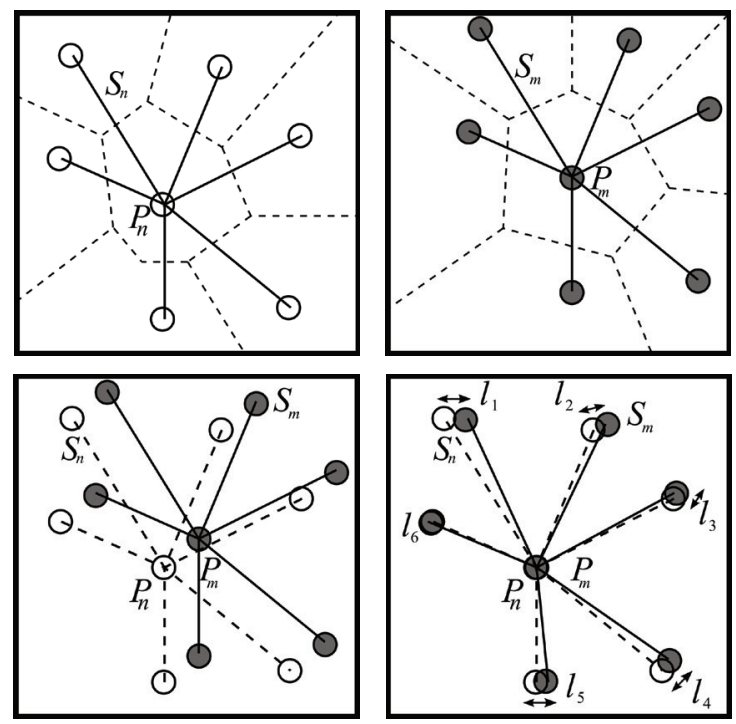

Figura 3. Tesselação de Voronoï para dois instantes de tempos consecutivos e indicação das respetivas estrelas de Voronoï e a determinação do deslocamento (Aleixo, 2013).

Comparativamente a outros algoritmos de PTV este algoritmo provou ser mais robusto (Capart et al., 2002), nomeadamente porque o algoritmo de correspondência, baseado na distância entre as extremidades da estrela de Voronoï é mais insensível à perda de partículas entre imagens consecutivas.

\section{A COMBINAÇÃO PIV-PTV}

Dadas as complementaridades entre ambas as técnicas, surge naturalmente a tentativa de as combinar aproveitar o melhor de cada, em particular no que se aplica a escoamentos bifásicos, por exemplo, água e sedimentos. Assim, a combinação desta técnica permitirá idealmente utilizar PIV onde a concentração de partículas é elevada e utilizar PTV onde a concentração de partículas é reduzida. Por outro lado, permite estender a resolução espacial da PIV utilizando para o efeito a PTV (Stitou e Riethmuller, 2001). Igualmente a técnica PTV pode tornar-se mais eficiente utilizando a PIV como primeira estimativa do deslocamento das partículas individuais. Esquematicamente a combinação PIVPTV é representada no fluxograma da Figura 4.

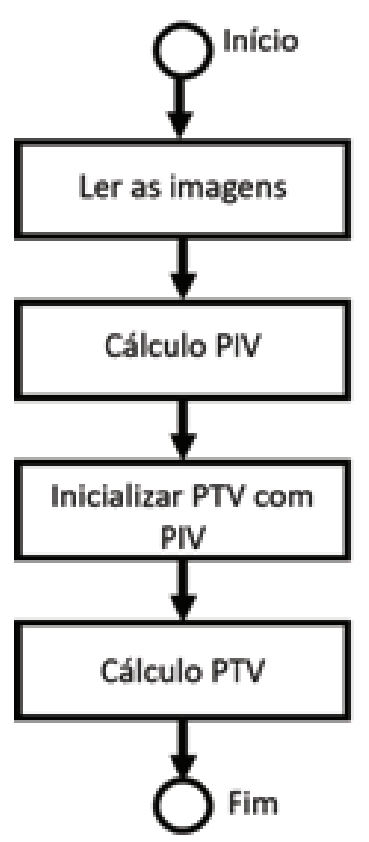

Figura 4. Fluxograma para a implementação da combinação PIV-PTV.

\section{CASO DE ESTUDO}

Utilizaram-se imagens provenientes de um escoamento de rotura de barragens para testar a aplicação desta técnica. As experiências de rotura de barragem foram realizadas no laboratório de hidráulica do Institute of Mechanics Materials and Civil Engineering da Université catholique de Louvain, Bélgica.

As medições foram realizadas num canal com 6 $\mathrm{m}$ de comprimento, 0,25 $\mathrm{m}$ de largura e $0,5 \mathrm{~m}$ de altura. A meio do canal uma comporta ligada a um veio pneumático divide o canal em duas partes iguais. Uma das partes é usada como reservatório. A comporta move-se verticalmente para baixo, sendo completamente removida num intervalo de tempo de cerca de 120 ms, permitindo a sua remoção ser considerada como instantânea (Lauber e Hager, 1998). Este canal é descrito em Spinewine e Zech (2007) e foi usado em diversos estudos de rutura de barragem como por exemplo Aleixo et al. (2010), Aleixo (2013), Aleixo et al. (2018). Um esquema do canal é apresentado na Figura 5. 


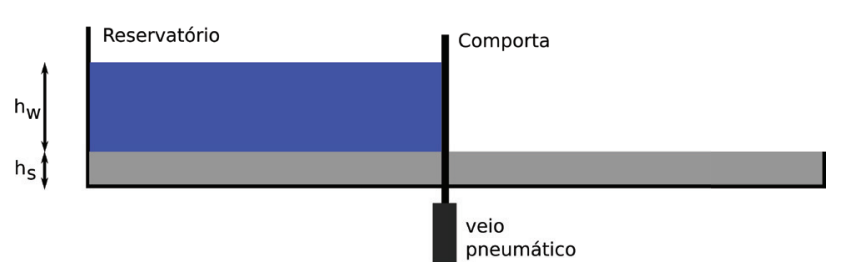

Figura 5. Esquema do canal de rutura de barragem do laboratório de hidráulica do Institute of Mechanics,

Materials and Civil Engineering. As variáveis $h_{w} \mathrm{e}$

$h_{w}$ representam a altura de água e de sedimentos respetivamente.

O escoamento de rotura de barragem foi realizado considerando um leito de sedimentos móveis, com uma altura de $0,10 \mathrm{~m}$ ao longo de toda a extensão do canal. $\mathrm{O}$ reservatório foi cheio até atingir uma cota de $0,325 \mathrm{~m}$ de água acima do leito de sedimentos, como exemplificado na Figura 5.

Os sedimentos utilizados foram pellets de PVC com uma densidade $s=1,63$ e um diâmetro médio de $d=3,85 \times 10^{-3} \mathrm{~m}$ (Figura 6). Para descrever o movimento da água, partículas de pliolite $(s=1.03)$ foram adicionadas no reservatório.

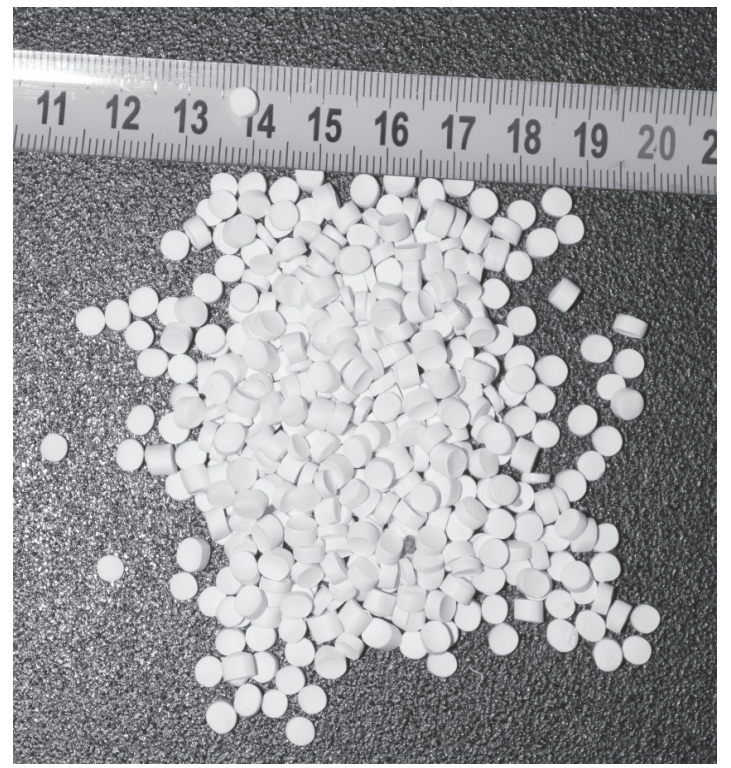

Figura 6. Pellets em PVC utilizados para modelar o leito móvel.

As imagens do escoamento foram adquiridas com uma máquina de aquisição rápida Photron equipada com uma lente Carl Zeiss de $50 \mathrm{~mm}$. A frequência de aquisição da câmara foi imposta a 1000 imagens por segundo. A limitação do buffer de memória limitou a aquisição de imagens a $2 \mathrm{~s}$, suficientes para capturar os instantes iniciais da rutura de barragem.

\section{RESULTADOS}

\subsection{Imagens do escoamento}

$\mathrm{Na}$ Figura 7 mostram-se algumas imagens do escoamento de rotura de barragem em diferentes instantes de tempo. Nestas imagens é possível ver o carácter fortemente transiente do escoamento, assim como as variações da superfície livre e da interface água-sedimentos.
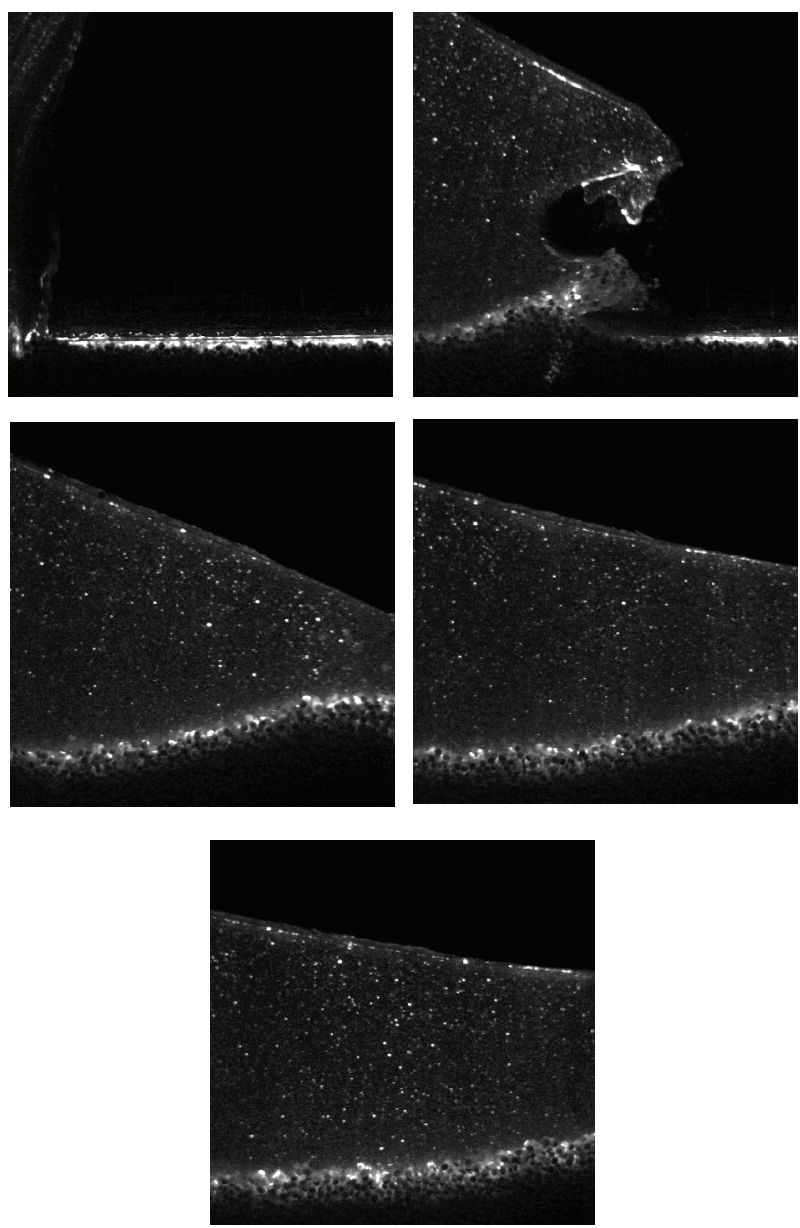

Figura 7. Diferentes instantes da rutura de barragem: $t=0 \mathrm{~s}, t=0.1 \mathrm{~s}, t=0.2 \mathrm{~s}, t=0.3 \mathrm{~s}$ e $t=0.4$. s.

\subsection{Resultados de PIV}

Utilizando a toolbox Matlab MatPIV 1.7 determinaram-se os campos de velocidade do escoamento de rotura de barragem para os instantes assinalados anteriormente. $\mathrm{O}$ processamento foi realizado utilizando um processo iterativo começando com áreas de interrogação de $128 \mathrm{px} \times 128 \mathrm{px}$ até uma área de interrogação de 32 px $\times 32$ px com 50\% de sobreposição entre áreas de interrogação. 

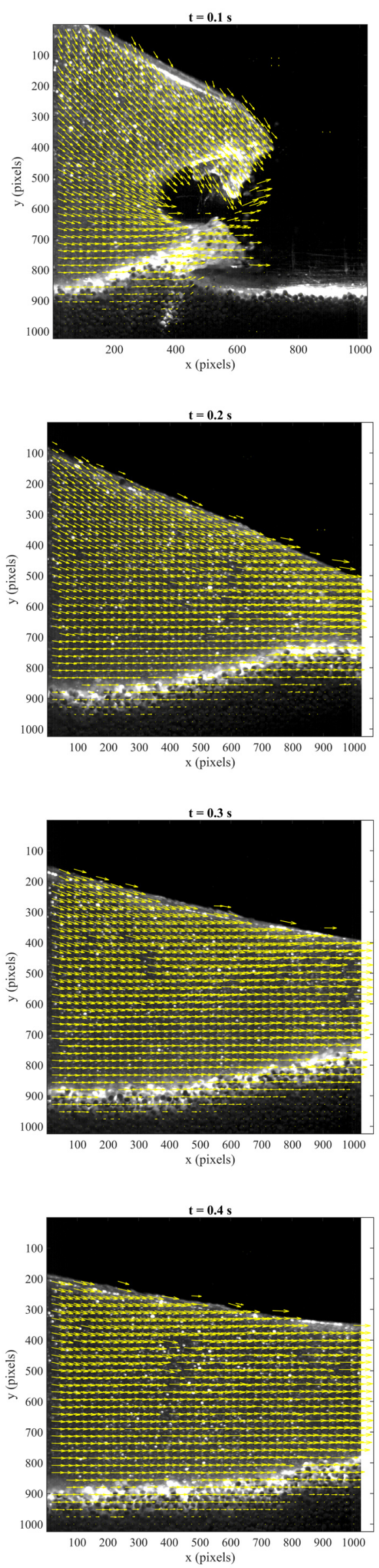

Figura 8. Resultado da aplicação da técnica PIV para diferentes instantes: $t=0.1 \mathrm{~s}, t=0.2 \mathrm{~s}, t=0.3 \mathrm{~s}$ e $t=0.4 \mathrm{~s}$.

\subsection{Resultados de PTV}

Uma região retangular junto ao leito foi selecionada para a aplicação do algoritmo de PTV baseado na tesselação de Voronoï. Os resultados são ilustrados na Figura 9. Da análise das figuras notase que a iluminação do leito é um ponto crítico. Uma iluminação pouco uniforme, como é o caso, complica o processo de deteção de partículas em camadas inferiores do leito utilizando métodos baseados na intensidade luminosa e diâmetro.
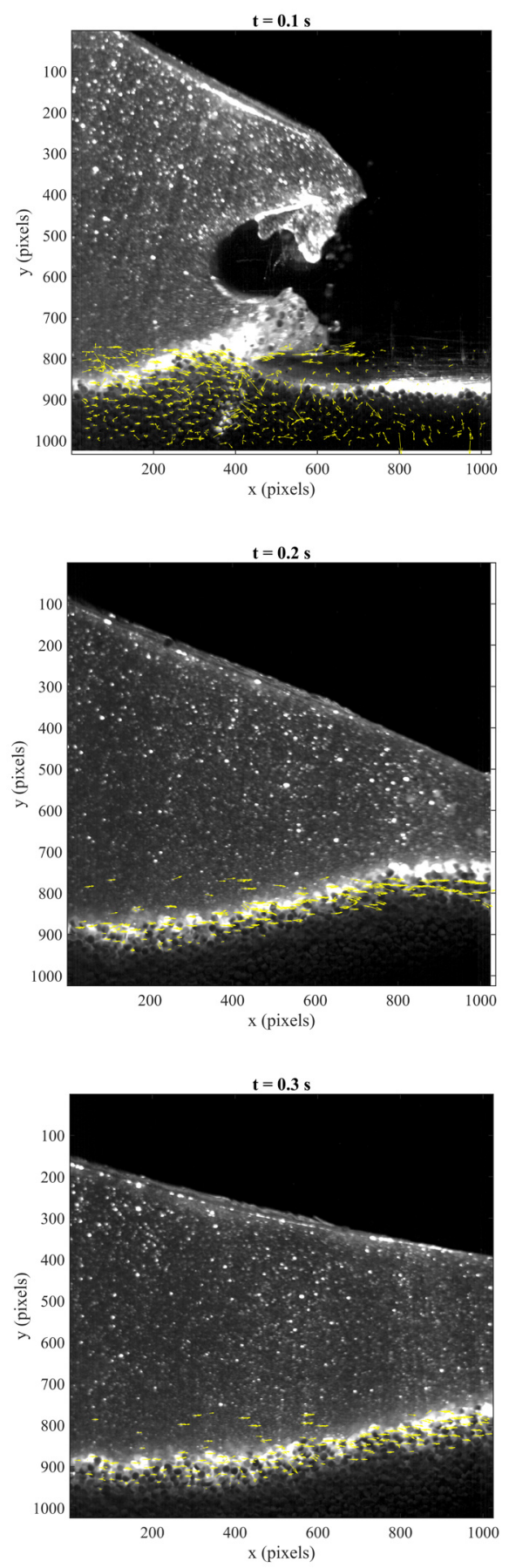

Figura 9. Resultado da aplicação da técnica PTV a uma região junto ao leito para diferentes instantes: $t=0.1 \mathrm{~s}$, $t=0.2 \mathrm{~s}, t=0.3 \mathrm{se} t=0.4 \mathrm{~s}$. 


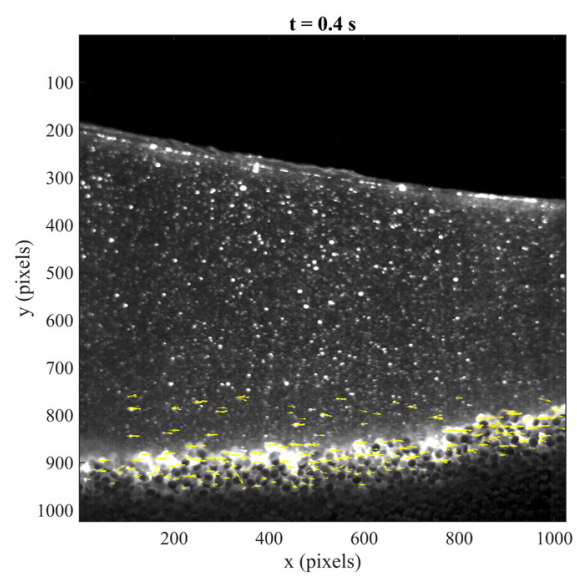

Figura 9. Resultado da aplicação da técnica PTV a uma região junto ao leito para diferentes instantes: $t=0.1 \mathrm{~s}$,

$$
t=0.2 \mathrm{~s}, t=0.3 \mathrm{~s} \text { e } t=0.4 \mathrm{~s} .
$$

\subsection{Resultados da combinação PIV-PTV}

Os perfis da Figura 10 mostram a comparação direta entre os resultados da PIV e da PTV. Como é possível ver, as duas técnicas mostram resultados compatíveis entre si. Contudo, para uma análise mais correta do leito de sedimentos, aplicou-se a combinação de ambas as técnicas. Os resultados são ilustrados na Figura 11.
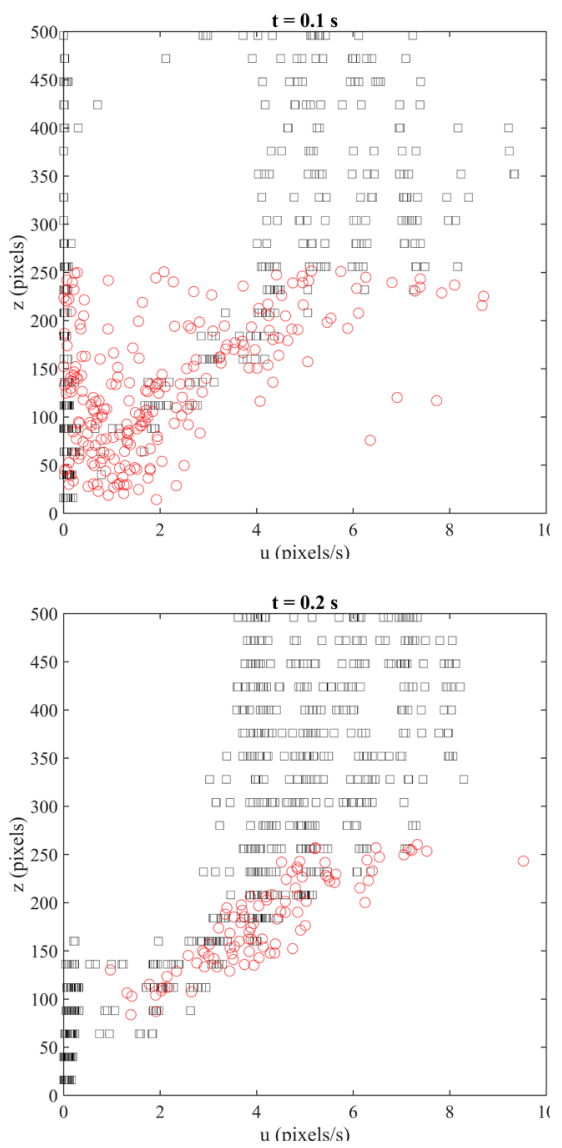

Figura 10. Comparação dos perfis de velocidade obtidos com PIV e PTV em diferentes instantes: $t=0 \mathrm{~s}$, $t=0.1 \mathrm{~s}, t=0.2 \mathrm{~s}, t=0.3 \mathrm{~s}$ e $t=0.4 \mathrm{~s}$.
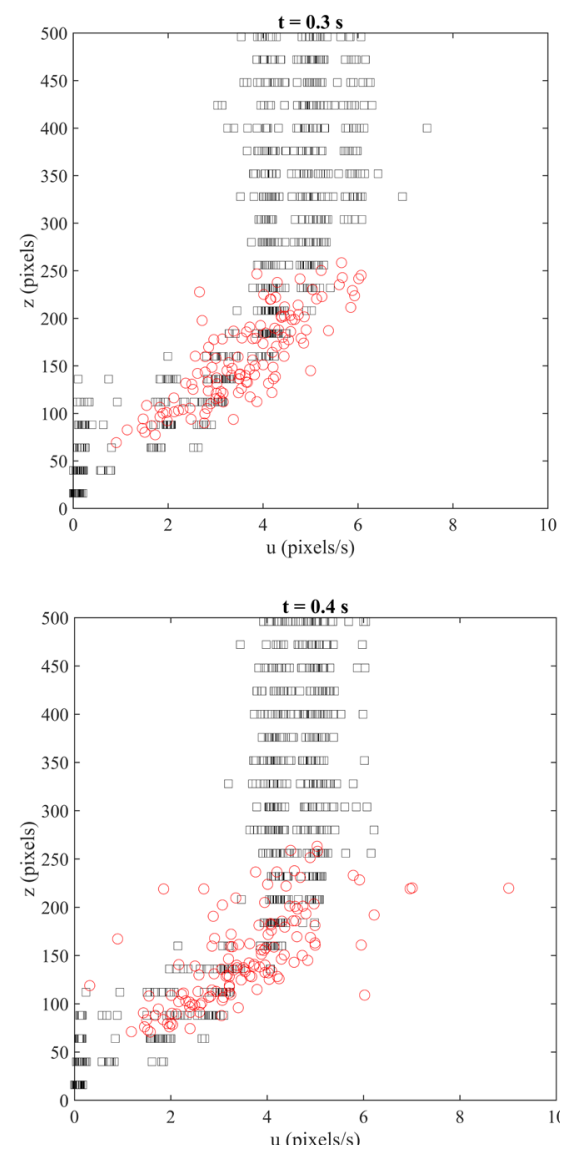

Figura 10. Comparação dos perfis de velocidade obtidos com PIV e PTV em diferentes instantes: $t=0 \mathrm{~s}, t=0.1 \mathrm{~s}, t=0.2 \mathrm{~s}, t=0.3 \mathrm{se} t=0.4 \mathrm{~s}$.
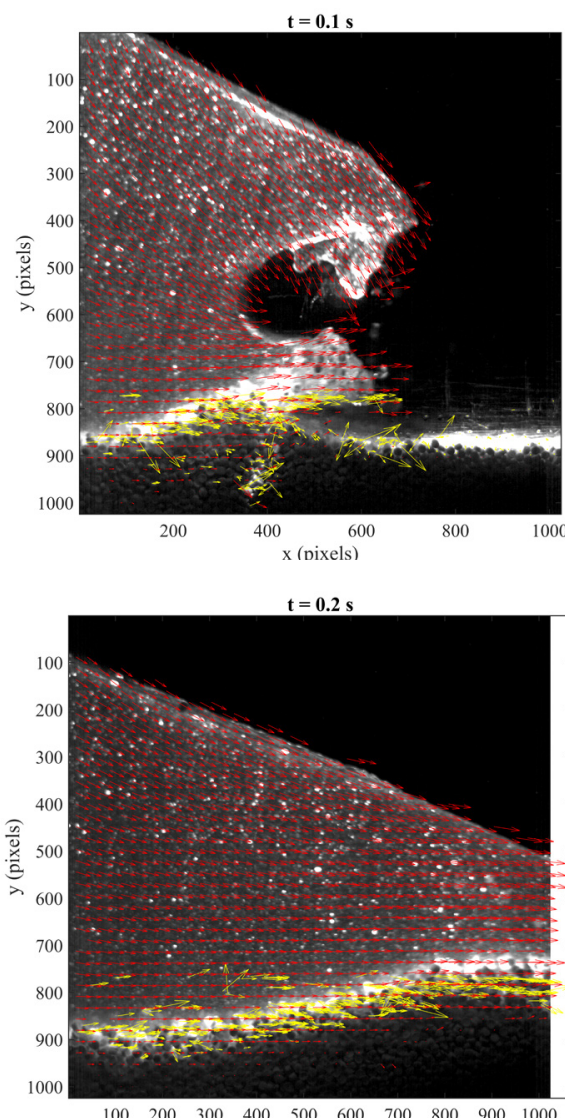

$\begin{array}{llllllllll}100 & 200 & 300 & 400 & 500 & 600 & 700 & 800 & 900 & 1000\end{array}$

Figura 11. Resultado do campo de velocidades medido com PIV-PTV em diferentes instantes: $t=0 \mathrm{~s}, t=0.1 \mathrm{~s}, t=0.2 \mathrm{~s}, t=0.3 \mathrm{~s} \mathrm{e} t=0.4 \mathrm{~s}$. 

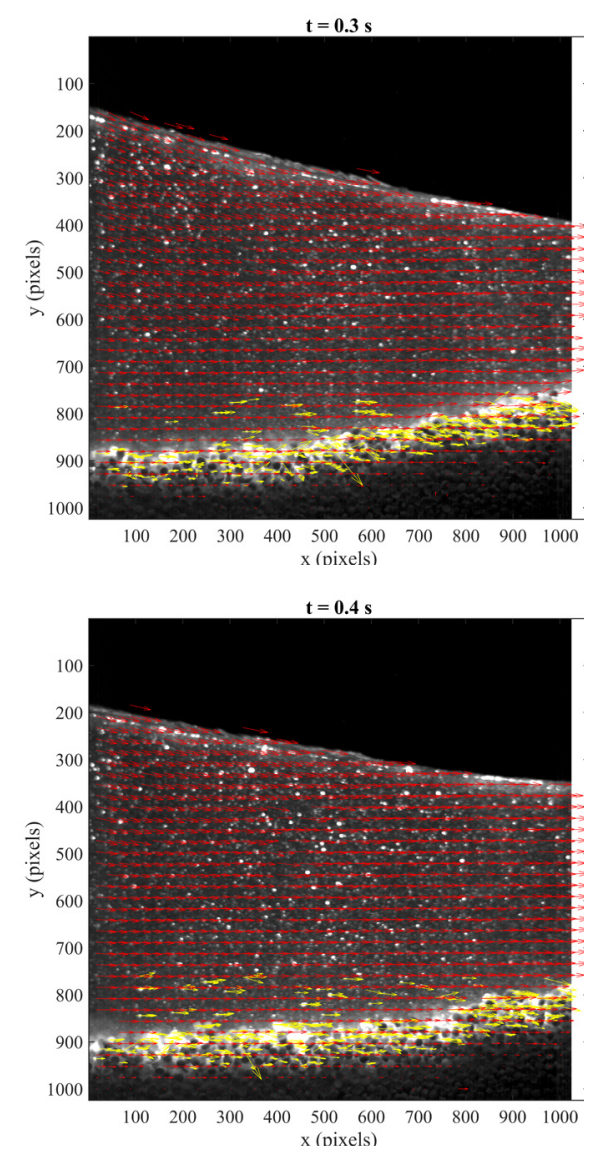

Figura 11. Resultado do campo de velocidades medido com PIV-PTV em diferentes instantes: $t=0 \mathrm{~s}, t=0.1 \mathrm{~s}$, $t=0.2 \mathrm{~s}, t=0.3 \mathrm{~s}$ e $t=0.4 \mathrm{~s}$.

\section{CONCLUSÕES}

Apresentou-se neste artigo uma técnica combinada entre PIV e PTV. A vantagem desta técnica é contribuir para um aumento da resolução espacial da técnica PIV, aproveitando o ganho de resolução espacial trazido pela PTV, ao mesmo tempo que o processo de seguimento de partículas da fase sólida se torna mais eficiente, usando como estimativa o campo de velocidades determinado pela técnica PIV.

A iluminação do leito de sedimentos deve ser igualmente uniforme para uma melhor deteção das partículas e conseguinte seguimento.

A determinação de um procedimento de máscara automático, isto é, um procedimento que permita reconhecer as diferentes regiões do escoamento: i) sem escoamento, ii) água e iii) sedimentos poderá contribuir para uma melhoria da eficiência do método aqui apresentado.

\section{AGRADECIMENTOS}

Este trabalho teve o apoio financeiro do Projeto PTDC/ ECM-HID/6387/2014 - POCl-01-0145- FEDER-016825
- financiado por fundos do FEDER através do COMPETE2020-ProgramaOperacionalCompetitividade e Internacionalização (POCl) e por fundos nacionais através da FCT - Fundação para a Ciência e a Tecnologia, I.P.

\section{REFERÊNCIAS}

Adrian, R. J. (2005). Twenty years of particle image velocimetry. Experiments in Fluids 39, pp. 159-169.

Adrian, R. J. e Westerweel, J. (2011). Particle Image Velocimetry. Cambridge University Press.

Aleixo, R. (2013). Experimental study of the early stages of a dam-break flow over fixed and mobile beds. Tese de Doutoramento. Université catholique de Louvain. Bélgica

Aleixo, R., Soares-Frazão, S. e Zech, Y. (2010). Velocityfield measurements in a dam-break flow using a PTV Voronoï imaging technique. Experiments in Fluids 50:6, 1633-1649.

Aleixo, R., Soares-Frazão, S. e Zech, Y. (2018). Statistical analysis methods for transient flows - the dam-break case. DOI:10.1080/00221686.2018.1516700.

Capart, H., D. Young, and Y. Zech (2002). Voronoï imaging methods for the measurement of granular flows. Experiments in Fluids 32, pp. 121-135.

Keane R.D. e Adrian R.J. (1992). Theory of crosscorrelation analysis of PIV images. Appl Sci Res 49 pp. $191-215$

Lauber, G. e Hager, W.H. (1998). Experiments to dambreak wave: horizontal channel. Journal of Hydraulic Research, 36, 291-307.

Meynart, R. (1982). Digital image processing for speckle flow velocimetry. Rev. Sci. Instrum. 53. Pp. 110-111

Raffel, M., Willert, C., Werely, S., Kompenhans, J. (2007). Particle Image Velocimetry - A practical guide 2nd Edition. Springer.

Spinewine B. e Zech, Y. (2007). Small-scale laboratory dam-break waves on movable beds, Journal of Hydraulic Research Vol. 45 Extra Issue, pp. 73-86.

Scarano, F. e Riethmuller M. (2000). Advances in iterative multigrid PIV image processing. Experiments in Fluids 29, pp. S051-S060.

Stitou, A. e Riethmuller M. (2001). Extension of PIV to superresolution using PTV. Measurements Science Technology 13, pp. 1398-1403. 